22 IMPACT OF THE CARDIFF UNIVERSITY MSC PROGRAMME IN PALLIATIVE MEDICINE (10 YEARS IN INDIA)

Nikki Pease, Jo Hayes, Fiona Rawlinson, Ilora Finlay. Cardiff University, Velindre NHS Trust

10.1136/bmjspcare-2018-ASPabstracts.49

Background Since its inception in 1987, undertaking the 'Cardiff Diploma/MSc' has helped develop many Palliative Medicine specialists, teachers, service leaders and researchers both within the UK and Internationally. With annual financial support, first awarded in 2006, from the Commonwealth Scholarship Commission, doctors from India have been supported to undertake the Cardiff University MSc in Palliative Medicine.

Objectives This survey looks to quantify the impact of undertaking the Cardiff University Palliative Medicine Diploma or MSc on Indian doctors' practice.

Method All Indian alumni were invited to complete a short, 10-question, anonymous on line survey. Response rate was $61 \%(n=37)$. Respondents were asked to score $(0-100)$ the impact the MSc had on their clinical, teaching, management/ service lead, and research roles. As a measure of impact respondents were also invited to give details of any publications and special interests subsequently developed.

Results On a scale of $0-100$ (zero to high impact) average collated self-scored impact of undertaking the MSc in Palliative Medicine were; clinical role $82 \%(50-100)$; teaching role $82 \%$ (30-100); management/service lead role 68\% (20-100) and research role $74 \%$ (35-100).

Over 50 journal publications, 20 International poster presentations and several conference workshops were listed as a result of MSc work.

$70 \%$ of respondents had developed a special interest as a result of undertaking the Diploma/MSc.

Conclusion 10 years on it would seem that the Cardiff University MSc has had significant impact. Future plans include collaboration with the India alumni, to roll out further teaching and training material and to develop international research between the Cardiff team and India.

\section{OBTAINING ETHICAL APPROVAL FOR PALLIATIVE MEDICINE MASTERS RESEARCH - WHAT ARE THE CHALLENGES?}

Anthony Williams, Isobel Jackson, Fiona Rawlinson. Velindre NHS Trust, Cardiff University

\subsection{6/bmjspcare-2018-ASPabstracts.50}

Background There is a growing focus on research in Palliative Medicine but the challenges in undertaking research in this area are significant. One of these challenges is often perceived to be the process of obtaining ethical approval from a Research Ethics Committee (REC). The aim of this project was to review the published literature relating to the research ethics process within palliative medicine and relate that to qualitative and quantitative data on this process from students undertaking a Palliative Medicine MSc at Cardiff University.

Methods A literature search was conducted through an OVID database search and hand-searching relevant journal articles. This identified seven articles which were reviewed in order to provide a qualitative assessment of the literature. A semi-structured online questionnaire was designed and distributed via email to all previous palliative medicine MSc candidates at
Cardiff University from 2010-2015. Qualitative data was reviewed by hand to explore common themes within the replies.

Results A common theme identified from the published literature was a perception that many RECs take a cautious approach to the palliative patient population. Of the 129 alumni, 114 were contacted and 34 replied. The student's feedback highlighted that some RECs are unfamiliar with qualitative research and the process is time-consuming. However, $85 \%$ of the students surveyed stated that they obtained approval without significant change to the project.

Conclusion This study highlights some of the barriers to research in palliative medicine and shows that students are still faced with variable insight into the nature of this research from RECs. Further study in this area is important to ensure that necessary research within palliative medicine is not avoided due to barriers in the ethical approval process. A strong palliative medicine presence within RECs may be beneficial to the appropriate assessment of research within this field.

\section{WHAT CONCERNS DO MEDICAL STUDENTS HAVE WHEN FACED WITH CARING FOR A DYING PATIENT?}

Ben Dietsch, Jane Wale, Zana Saunders. Willen Hospice, Milton Keynes University Hospital, University of Buckingham Medical School, Open University

\subsection{6/bmjspcare-2018-ASPabstracts. 51}

Background Existing research suggests that:

- Students are highly interested in palliative care competencies and appreciate formal curriculum covering competencies, in conjunction with opportunity to learn skills experientially.

- Palliative care encompasses a range of skills and knowledge which can potentially be addressed in a variety of ways (eg through lectures, SSMs, communication skills and reflection).

- Palliative care curricula for undergraduate medical students could creatively cover this breadth of knowledge/skills using learning modalities.

However, curriculum tend to be developed based upon the knowledge and skills that professionals feel students should have. There is little research that considers the specific concerns and learning needs identified by medical students themselves, in response to open questioning.

Methods Third year medical students, were asked to reflect on their experience of seeing dying patients, both on the wards and in the media and to answer the open question 'What concerns do you have about caring for a dying patient?' Their responses were collated and analysed for themes.

Results Medical students identified a range of concerns and potential learning needs. These included knowledge based competencies (such as managing of pain and symptoms; 20.8\%); ethical and legal issues (33.3\%) but mostly a desire to provide patient-centred care, respecting the patient's wishes and autonomy (41.7\%). 20.8\% of students identified their own personal concerns when caring for the dying, Interestingly, an explicit concern about communication was raised by just $12.5 \%$, but communication issues were implicit in $37.5 \%$ responses.

Conclusions In response to an open question, third year medical students identified insightful concerns about caring for the 